\title{
The central star of the planetary nebula PB 8: a Wolf-Rayet-type wind of an unusual WN/WC chemical composition ${ }^{\star} \star \star \star$
}

\author{
H. Todt ${ }^{1}$, M. Peña ${ }^{2}$, W.-R. Hamann ${ }^{1}$, and G. Gräfener ${ }^{1,3}$ \\ 1 University of Potsdam, Institute for Physics and Astronomy, 14476 Potsdam, Germany \\ e-mail: htodt@astro.physik.uni-potsdam.de \\ 2 Instituto de Astronomía, Universidad Nacional Autónoma de México, Apdo. Postal 70264, México D.F. 04510, México \\ 3 Armagh Observatory, College Hill, Armagh BT61 9DG, Northern Ireland
}

Received 25 March 2009 / Accepted 8 March 20100

\section{ABSTRACT}

\begin{abstract}
A considerable fraction of the central stars of planetary nebulæ (CSPNe) are hydrogen-deficient. As a rule, these CSPNe exhibit a chemical composition of helium, carbon, and oxygen with the majority showing Wolf-Rayet-like emission line spectra. These stars are classified as CSPNe of a spectral type [WC]. We perform a spectral analysis of CSPN PB 8 with the Potsdam Wolf-Rayet (PoWR) models for expanding atmospheres. The source PB 8 displays wind-broadened emission lines from strong mass loss. Most strikingly, we find that its surface composition is hydrogen-deficient, but not carbon-rich. With mass fractions of 55\% helium, 40\% hydrogen, $1.3 \%$ carbon, $2 \%$ nitrogen, and $1.3 \%$ oxygen, it differs greatly from the $30-50 \%$ of carbon which are typically seen in [WC]-type central stars. The atmospheric mixture in PB 8 has an analogy in the WN/WC transition type among the massive Wolf-Rayet stars. Therefore we suggest to introduce a new spectral type [WN/WC] for CSPNe, with PB 8 as its first member. The central star of PB 8 has a relatively low temperature of $T_{*}=52 \mathrm{kK}$, as expected for central stars in their early evolutionary stages. Its surrounding nebula is less than 3000 years old, i.e. relatively young. Existing calculations for the post-AGB evolution can produce hydrogen-deficient stars of the [WC] type, but do not predict the composition found in PB 8. We discuss various scenarios that might explain the origin of this unique object.
\end{abstract}

Key words. stars: abundances - stars: AGB and post-AGB - stars: atmospheres - stars: mass-loss - stars: individual: PN PB 8 stars: Wolf-Rayet

\section{Introduction}

A planetary nebula (PN) surrounds a central star which is hot enough $\left(T_{*}>25000 \mathrm{~K}\right)$ to ionize its circumstellar matter. According to the well-established scenario (e.g. Paczyński 1970; Schönberner 1989), the central star of the planetary nebula (CSPN) ejects its nebula while suffering thermal pulses at the tip of the asymptotic giant branch (AGB). In the subsequent PN phase it evolves rapidly towards the white dwarf cooling sequence.

Most of the CSPNe show a hydrogen-rich surface composition. Among the Galactic central stars, 5-6\% are hydrogendeficient and show emission lines in their spectra (Tylenda et al. 1993; Acker \& Neiner 2003). Moreover, half of these hydrogendeficient stars have spectra similar to those of massive WolfRayet stars of the carbon sequence and are therefore classified as spectral type [WC]. They have a strong stellar wind composed of helium, carbon, and oxygen. Typical carbon surface-abundances have been found to lie between $30 \%$ and $50 \%$ by mass (see the reviews by Koesterke 2001; Crowther 2008).

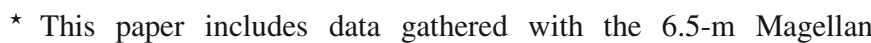
Telescopes located at Las Campanas Observatory, Chile.

$\star \star$ Some of the data presented in this paper were obtained from the Multimission Archive at the Space Telescope Science Institute (MAST). STScI is operated by the AURA, Inc., under NASA contract NAS5-26555. Support for MAST for non-HST data is provided mainly by the NASA Office of Space Science via grant NAG5-7584. Based on INES data from the IUE satellite.
The central star (CS) of the planetary nebula PB 8 (PN G292.4+04.1) was first classified by Méndez (1991) as a hydrogen-rich Of-WR(H) star due to the $\mathrm{H} \gamma \mathrm{P}$ Cygni profile and the appearance of an unusually strong He II 4686 emission line.

In contrast, Acker \& Neiner (2003) classified this star as a [WC5-6] type star.

Below we analyze optical, IUE, and FUSE spectra of the central star PB 8 by means of the Potsdam Wolf-Rayet (PoWR) model atmosphere code. The observations are introduced in Sect. 2. Spectral modeling is briefly explained in Sect. 3. In Sect. 4 we describe the spectral analysis, and the results are discussed in the final section (Sect. 5).

\section{Observations}

\subsection{Optical spectrum}

High-resolution spectroscopy of PB 8 was performed on 2006 May 9 at Las Campanas Observatory (Carnegie Institution) with the Clay $6.5 \mathrm{~m}$-telescope and the double échelle spectrograph MIKE (Magellan Inamori Kyocera Echelle). This spectrograph operates with two arms, which allow the observer to obtain blue and red spectra simultaneously. The standard grating settings provided wavelength coverage of 3350-5050 $\mathrm{A}$ for the blue and 4950-9400 $\AA$ for the red. Three spectra with exposure times of $300 \mathrm{~s}, 600 \mathrm{~s}$ and $900 \mathrm{~s}$ were obtained. The slit width was $1^{\prime \prime}$ and was centered on the central star. A binning of $2 \times 2$ pixels was used, providing a plate scale of $0.26^{\prime \prime}$ per pixel. The spectral 
resolution varied from $0.14 \AA$ to $0.17 \AA$ in the blue and from $0.23 \AA$ to $0.27 \AA$ in the red as measured with the comparison lamp.

The data were reduced with standard procedures from the IRAF reduction packages ${ }^{1}$. Spectra were extracted with a $1.52^{\prime \prime}$ wide window and flux-calibrated with respect to standard stars. The three spectra were then weighted by exposure time and were finally combined.

\subsection{UV spectra}

A low-resolution UV spectrum (1200 to $2000 \AA$ ), taken with the International Ultraviolet Explorer (IUE) and a high-resolution FUV spectrum (960 to $1190 \AA$ ) , taken with the Far Ultraviolet Spectroscopic Explorer (FUSE), were retrieved from the MAST archive. For the UV range we used an exposure-time weighted combination of the IUE spectra SWP28434LL $\left(t_{\exp }=2400 \mathrm{~s}\right)$ and SWP30476LL $\left(t_{\exp }=7800 \mathrm{~s}\right)$, both taken with the "large" IUE aperture. The spectral resolution was about $5 \AA$, and the estimated S/N ratio is roughly 10 for $1200-1700 \AA$ and 20 for 1700-2000 A.

The FUSE observation of PB 8 was performed with the LWRS aperture of $30^{\prime \prime} \times 30^{\prime \prime}$ in run Z9111301000. We used a coadded spectrum "all4ttagfcal" from the CalFUSE pipeline, which is already rebinned to $0.1 \AA$ to improve the $\mathrm{S} / \mathrm{N}$ ratio, but is still sufficient to resolve interstellar $\mathrm{H}_{2}$ absorption lines.

\section{Methods}

\subsection{Spectral modeling}

For the spectral analysis we employed the PoWR models of expanding atmospheres. The PoWR code solves the non-LTE radiative transfer in a spherically expanding atmosphere simultaneously with the statistical equilibrium equations and accounts at the same time for energy conservation. Iron-group line blanketing is treated by means of the superlevel approach (Gräfener et al. 2002), and wind clumping in first-order approximation is taken into account (Hamann \& Gräfener 2004). We do not calculate hydrodynamically consistent models, but assume a velocity field following a $\beta$-law with $\beta=1$. Our present computations include complex atomic models for hydrogen, helium, carbon, oxygen, nitrogen, phosphorus, silicon, and the iron-group elements.

After the computation of the synthetic spectrum, the models need to be corrected for interstellar extinction. Dust extinction was taken into account by the reddening law of Cardelli et al. (1989). Interstellar line absorption in the FUSE range was calculated with the templates from McCandliss (2003) for $\mathrm{H}_{2}$, and Groenewegen \& Lamers (1989) for the Lyman series.

\subsection{Spectral fitting}

The typical emission-line spectra of Wolf-Rayet stars are predominantly formed by recombination processes in their dense stellar winds. Therefore the continuum-normalized spectrum shows a useful scale-invariance: for a given stellar temperature $T_{*}$ and chemical composition, the equivalent widths of the emission lines depend in first approximation only on the ratio between the volume emission measure of the wind and the area of

\footnotetext{
${ }^{1}$ IRAF is distributed by NOAO, which is operated by AURA, Inc., under contract to the National Science Foundation.
}

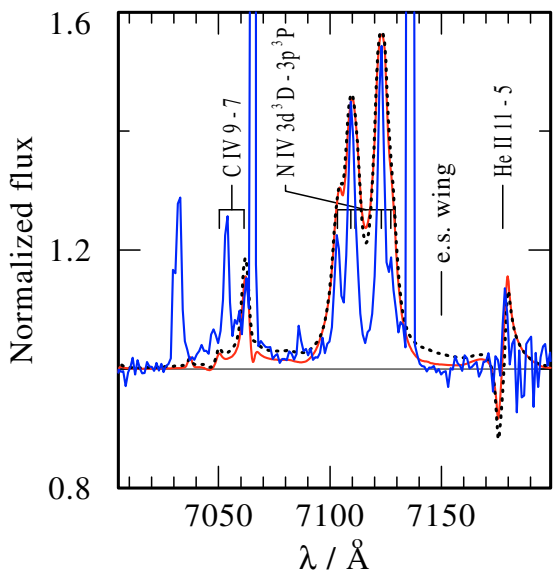

Fig. 1. Electron scattering (e.s.) wings for two similar models with same $R_{\mathrm{t}}$ but different density contrast $D$. The homogeneous model (black dotted line) predicts stronger e.s. wings than observed (blue solid). Despite the uncertainty due to normalization to the continuum, the model with $D=10$ (red solid) seems to be more consistent with the observation, although a higher clumping factor $D$ cannot be excluded. The observation is rebinned to $1 \AA$ for noise reduction.

the stellar surface. An equivalent quantity, which has been introduced by Schmutz et al. (1989), is the transformed radius

$R_{\mathrm{t}}=R_{*}\left[\frac{v_{\infty}}{2500 \mathrm{~km} \mathrm{~s}^{-1}} / \frac{\dot{M} \sqrt{D}}{10^{-4} M_{\odot} a^{-1}}\right]^{2 / 3}$.

Different combinations of stellar radii $R_{*}$ and mass-loss rates $\dot{M}$ can thus lead to the same emission-line strengths. In the form given here, the invariance also includes the micro-clumping parameter $D$, which is defined as the density contrast between wind clumps and a smooth wind of the same mass-loss rate. Consequently, mass-loss rates derived empirically from fitting the emission-line spectrum depend on the adopted value of $D$. The latter can be constrained by fitting the extended electron scattering wings of strong emission lines (e.g. Hamann \& Koesterke 1998).

\section{Analysis}

Models for an un-clumped wind $(D=1)$ predict e.s. wings to be stronger than observed. For the central star of PB 8 we find that $D=10$ is consistent with the observation (cf. Fig. 1).

For the terminal velocity $v_{\infty}$, we obtained a value of $1000 \mathrm{~km} \mathrm{~s}^{-1}$ from the width of the UV P-Cygni line profiles (cf. Fig. 2). Note that the width of the absorption profile is matched by our final model, but not the rather round shape of the profile. The observed shape of the absorption profile indicates a softer increase of the velocity in the outer parts of the stellar wind, as described, e.g., by a double- $\beta$ law (Hillier \& Miller 1999).

A corresponding synthetic spectrum, where $40 \%$ of the terminal velocity are attributed to a second, flatter $\beta$-law with $\beta_{2}=8$, is included in Fig. 2. From the limited agreement of the blue edges of the $\mathrm{P} v$ doublet, we estimate the uncertainty in $v_{\infty}$ to $\pm 100 \mathrm{~km} \mathrm{~s}^{-1}$.

Line broadening by microturbulence is also included in our models. From the shape of the line profiles we deduce a microturbulence velocity of less than $50 \mathrm{~km} \mathrm{~s}^{-1}$.

Stellar luminosity and mass were set to typical values for CSPNe, $L=6000 L_{\odot}$ and $M=0.6 M_{\odot}$ (see e.g. Schönberner et al. 2005b; Miller Bertolami \& Althaus 2007). The absolute 


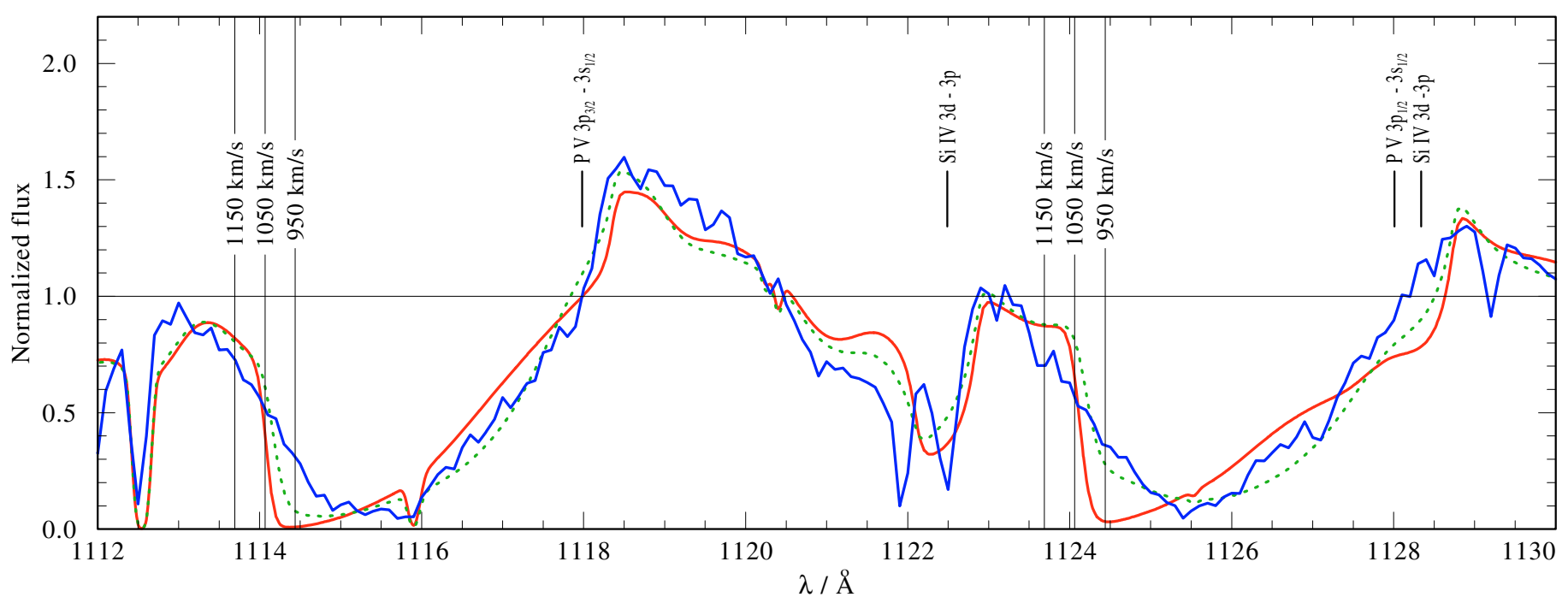

Fig. 2. Detail of the FUSE spectrum showing the PV resonance doublet, observation (blue line) vs. the final PoWR model (red solid line), including ISM absorption. The round shape of the absorption troughs is slightly better reproduced (green dashed line) when a double- $\beta$ law is adopted for the velocity field (see text).

flux of the model is diluted by its distance, which we consider to be a free parameter, as no certain distance is known. With the help of Eq. (1) the results can be easily scaled to a different luminosity. The value of $M$ has no noticeable influence on the synthetic spectra.

As aforementioned, the strength of WR emission lines mainly depends on the transformed radius $R_{\mathrm{t}}$, the stellar temperature $T_{*}$, and the chemical abundances.

We started our analysis by determining $R_{\mathrm{t}}$ and $T_{*}$ from a grid of models and then measured the abundances while the other two parameters were kept fixed.

Synthetic spectra from the grid of WNL model atmospheres ${ }^{2}$ (Hamann \& Gräfener 2004) were compared with the observed spectrum of PB 8, giving a first estimate of $\log T_{*} \approx 4.7$ and $\log R_{\mathrm{t}} / R_{\odot}=1.4$. From test calculations we obtained a first estimate of the chemical composition (see Table 2, model A). With these chemical abundances, an adopted luminosity of $L=$ $6000 L_{\odot}$, and a mass of $M=0.6 M_{\odot}$ we computed a refined grid of models around our first estimate $\left(T_{*}, R_{\mathrm{t}}\right)$.

For this grid, the line-strength ratios C IV 5800/C III 4650, N IV 7100/N III 4634, and He II 4686/He I 5876 were calculated and plotted as contour lines over the grid. The contour plot for N IV 7100/N III 4634 is shown in Fig. 3. Using line ratios instead of the absolute line strengths diminishes the influence of chemical abundances. Moreover, Fig. 3 shows that in the parameter range under consideration the line ratio depends almost only on the temperature and not on the transformed radius. In this way, the temperature determination de-couples from $R_{\mathrm{t}}$. The equivalent width of the C III 4650 line cannot be measured accurately, because this line is partly overlapping with the N III 4634 line (see Fig. 5). Therefore we used the peak height of the strongest multiplet component as a measure of the line strength, which is less affected by blending than the equivalent width. Peak ratios from observation and models are listed in Table 1. From our optical observation we estimate the uncertainty in the normalized continuum to be on the order of $10 \%$. We consider this to be the uncertainty in the peak measurement, which we then use to infer a $20 \%$ error in the measured ratio.

\footnotetext{
${ }^{2}$ http://www.astro.physik. uni-potsdam.de
}

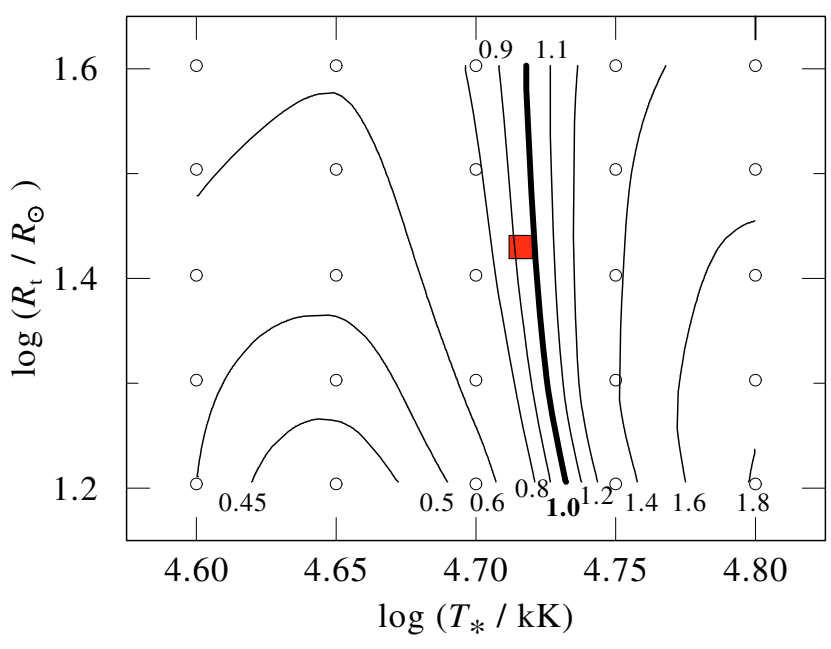

Fig. 3. Contours of the ratio between the peak heights of N IV 7100 to N III 4643. The thick contour represents the measured value. The open circles indicate the calculated models. Between these data points the contour lines are interpolated. The best-fitting model for PB 8 is indicated by the red square.

Table 1. Ratios between the peak heights: measured ratios, derived temperatures, and ratios from the final model (model B).

\begin{tabular}{llll}
\hline \hline & C IV/C III & N IV/N III & He II /He I \\
& $5800 / 4650$ & $7100 / 4634$ & $4686 / 5876$ \\
\hline Observed & $1.4 \pm 0.3$ & $1.0 \pm 0.2$ & $2.3 \pm 0.5$ \\
Derived $T_{*}$ & $50 \pm 4 \mathrm{kK}$ & $54 \pm 2 \mathrm{kK}$ & $52 \pm 3 \mathrm{kK}$ \\
Final model & 1.6 & 0.9 & 2.7 \\
\hline
\end{tabular}

As included in Table 1, slightly different stellar temperatures are derived from the different elements. But within the inferred uncertainties a temperature of $T_{*}=52 \mathrm{kK}$ is consistent with all three observed line ratios.

Although the line strengths of most of the spectral lines of carbon, oxygen, and nitrogen can be reproduced by our first estimate model A (Fig. 4) some of the unblended helium lines appear stronger than observed. 


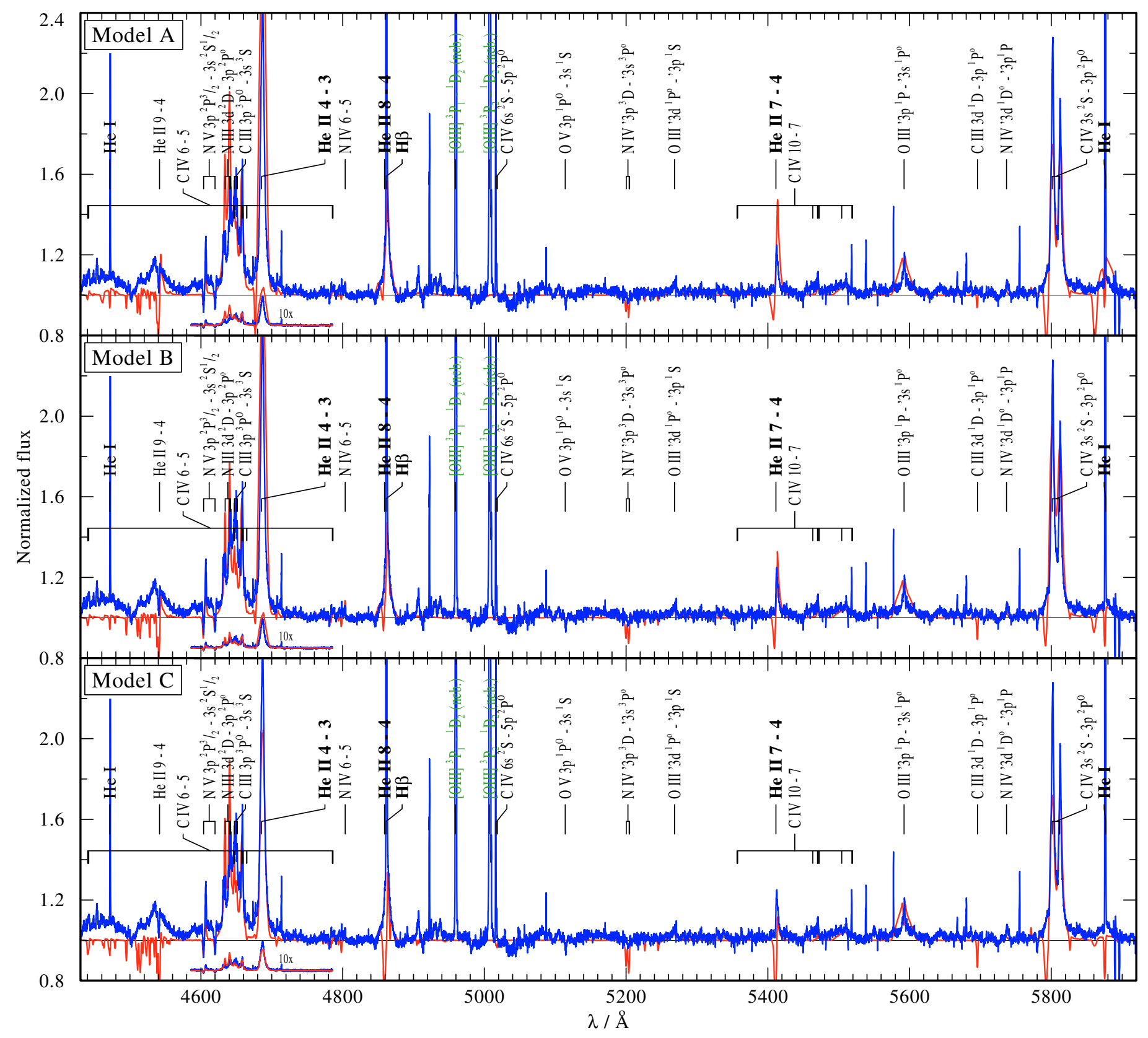

Fig. 4. PB 8: detail of the optical spectrum, comparison of the PoWR models A, B, and C (red lines) vs. observation (blue). PoWR model A (upper panel) with only $30 \%$ hydrogen. The emission lines from helium appear much too strong. PoWR model C (lower panel) with reduced mass-loss rate relative to model A and reduced helium abundance. To recover the strength of the emission lines of carbon, nitrogen, and oxygen of model A, their abundances are increased. The helium emission lines are weaker than observed. This model is considered as an upper limit for the hydrogen, carbon, oxygen, and nitrogen abundance, and as a lower limit for the mass-loss rate and the helium abundance. The compromise between models $\mathrm{A}$ and $\mathrm{C}$ and therefore our final model is model $\mathrm{B}$, shown in the second panel.

Therefore we calculated a model with the same $T_{*}$, but half the mass-loss rate $\left(2^{2 / 3} \times R_{\mathrm{t}}\right)$ and half the helium-mass fraction (model $\mathrm{C}$ in Table 2). To recover the line strengths of $\mathrm{H}, \mathrm{N}, \mathrm{O}$, and $\mathrm{C}$, the mass fraction for each of these elements is thus doubled.

Then, as expected, all of the spectral lines of helium are weaker than in model A (Fig. 4). The He II 4686 line in model $\mathrm{C}$ however seems to be more consistent with the observation. In contrast, the line blend of He II 4859 and $\mathrm{H} \beta$ exhibits deep absorption features, which are not observed. Furthermore, the modeled He I 5876 line is now much too weak in the model.

For our final fit we thus chose a model with parameter values in between those of model A and C. As a compromise, the best fit of all spectral lines is achieved with our final model B at a stellar temperature of $T_{*}=52 \mathrm{kK}$ and a transformed radius of $R_{\mathrm{t}}=26.9 R_{\odot}$ (cf. Figs. 5 and 6 ).

Parallel to the fitting of the normalized spectrum we obtained the synthetic spectral energy distribution (SED) in absolute units. This model-SED was fitted to the calibrated spectra and photometric measurements by adjusting the distance and the reddening parameter $E_{B-V}$ (see Fig. 7).

The best SED-fit was obtained with a color excess of $E_{B-V}=$ 0.41 and $R_{V}=4$ (Cardelli et al. 1989). This value is considerably higher than the value of $E_{B-V}=0.24$ derived for the nebula from the Balmer line decrement by García-Rojas et al. (2009) for the same observations, but with an adopted value of 
H. Todt et al.: The central star of the planetary nebula PB 8
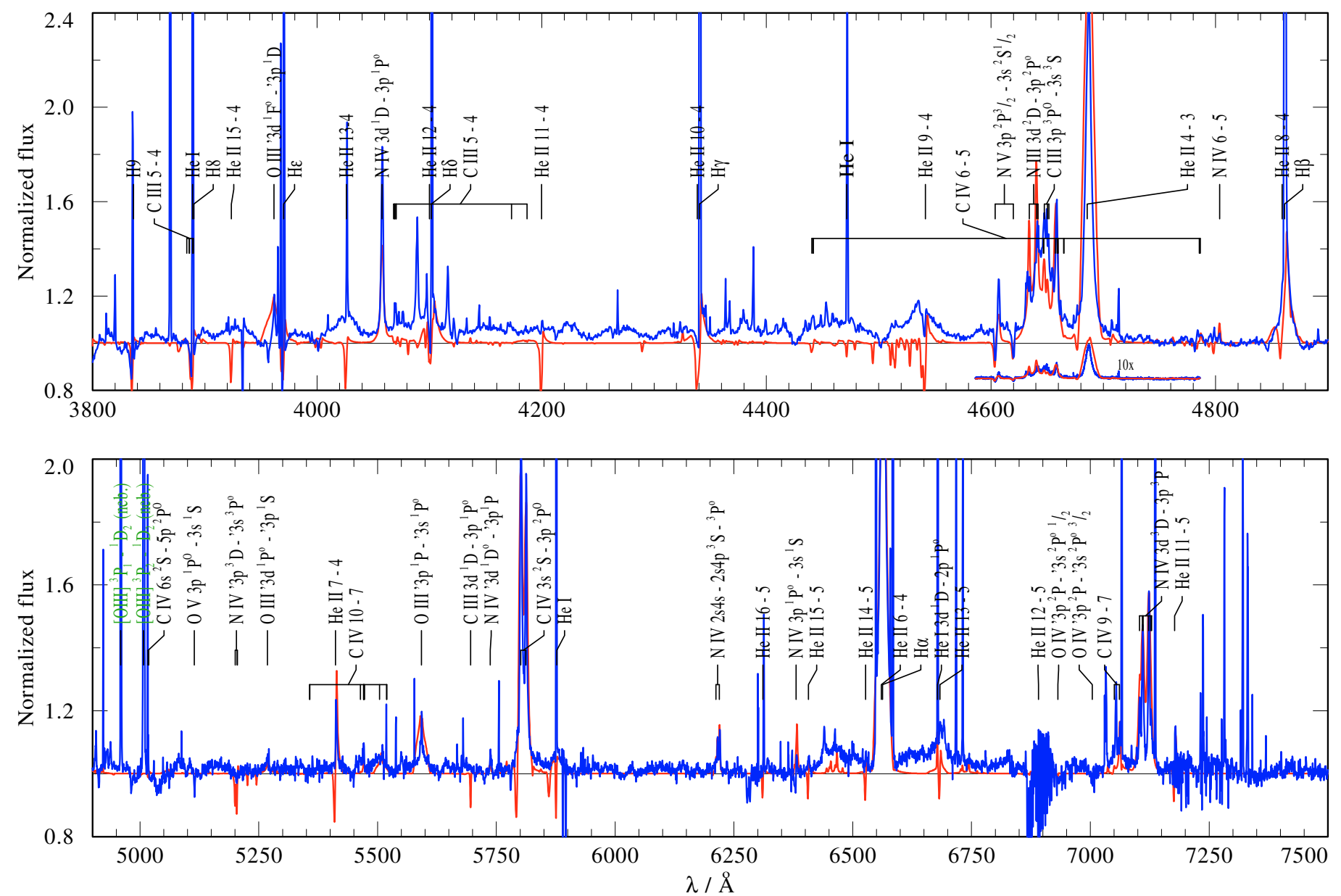

Fig. 5. Optical spectrum: observation of PB 8 (blue, thin line) and best-fitting PoWR model (red, thick line), both normalized to the model continuum. The observation is rebinned to $0.5 \AA$. The observed stellar spectrum is contaminated by the narrow nebular lines.

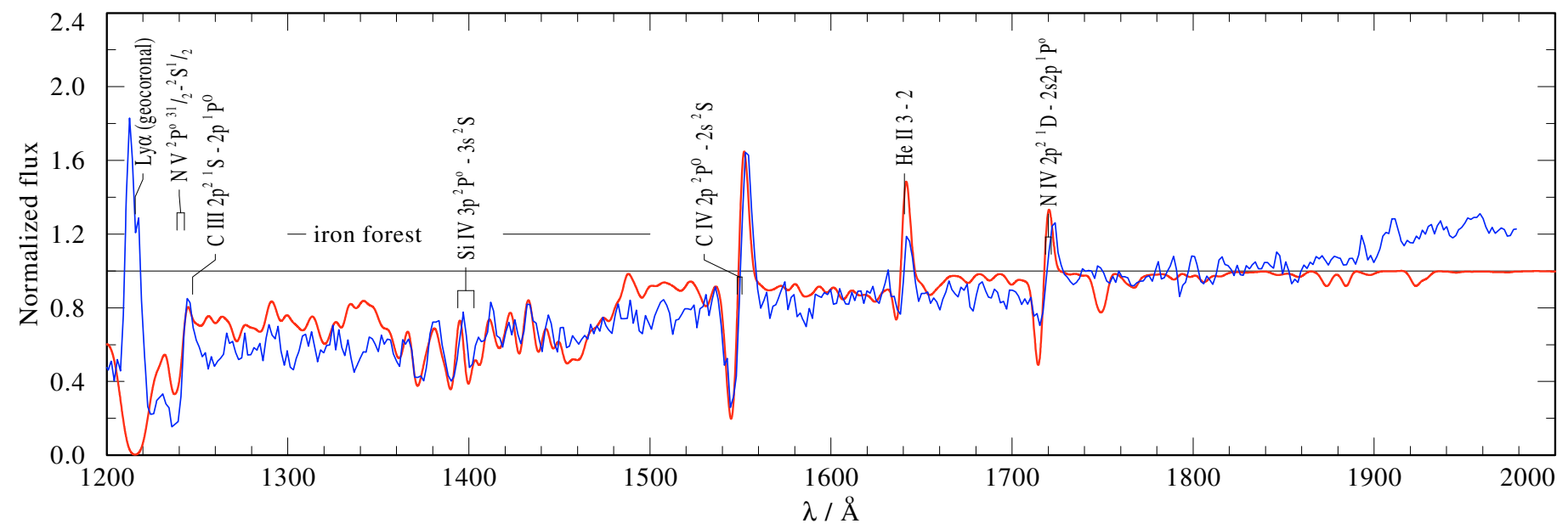

Fig. 6. Observed IUE spectrum of PB 8 (blue, thin line) vs. PoWR model (red, thick). The observed spectrum was normalized by the model continuum. The synthetic spectrum was folded with a Gaussian with a FWHM of $5 \AA$, corresponding to the spectral resolution of the IUE observation. The iron forest is only partially reproduced.

Table 2. Parameters for the PoWR models as shown in Figs. 4 and 5. Our final model is model B.

\begin{tabular}{|c|c|c|c|c|c|c|c|c|c|c|}
\hline model & $\begin{array}{c}\log R_{\mathrm{t}} \\
{\left[R_{\odot}\right]}\end{array}$ & $\begin{array}{c}\log \dot{M} \\
{\left[M_{\odot} \mathrm{a}^{-1}\right]}\end{array}$ & $\begin{array}{c}T_{*} \\
{[\mathrm{kK}]}\end{array}$ & $\mathrm{He}$ & $\begin{array}{c}\mathrm{H} \\
\% \mathrm{n}\end{array}$ & $\begin{array}{c}\mathrm{C} \\
\text { ass fra }\end{array}$ & $\begin{array}{c}\mathrm{N} \\
\text { ction }\end{array}$ & $\begin{array}{l}\mathrm{O} \\
- \\
\end{array}$ & $\begin{array}{c}d \\
{[\mathrm{kpc}]}\end{array}$ & $\begin{array}{c}E_{B-V} \\
{[\mathrm{mag}]}\end{array}$ \\
\hline $\mathrm{A}$ & 1.35 & -6.89 & 50 & 66 & 30 & 1 & 1.5 & 1 & 4.6 & 0.40 \\
\hline B & 1.43 & -7.07 & 52 & 55 & 40 & 1.3 & 2 & 1.3 & 4.2 & 0.41 \\
\hline C & 1.55 & -7.20 & 50 & 33 & 60 & 2 & 3 & 2 & 4.4 & 0.42 \\
\hline
\end{tabular}




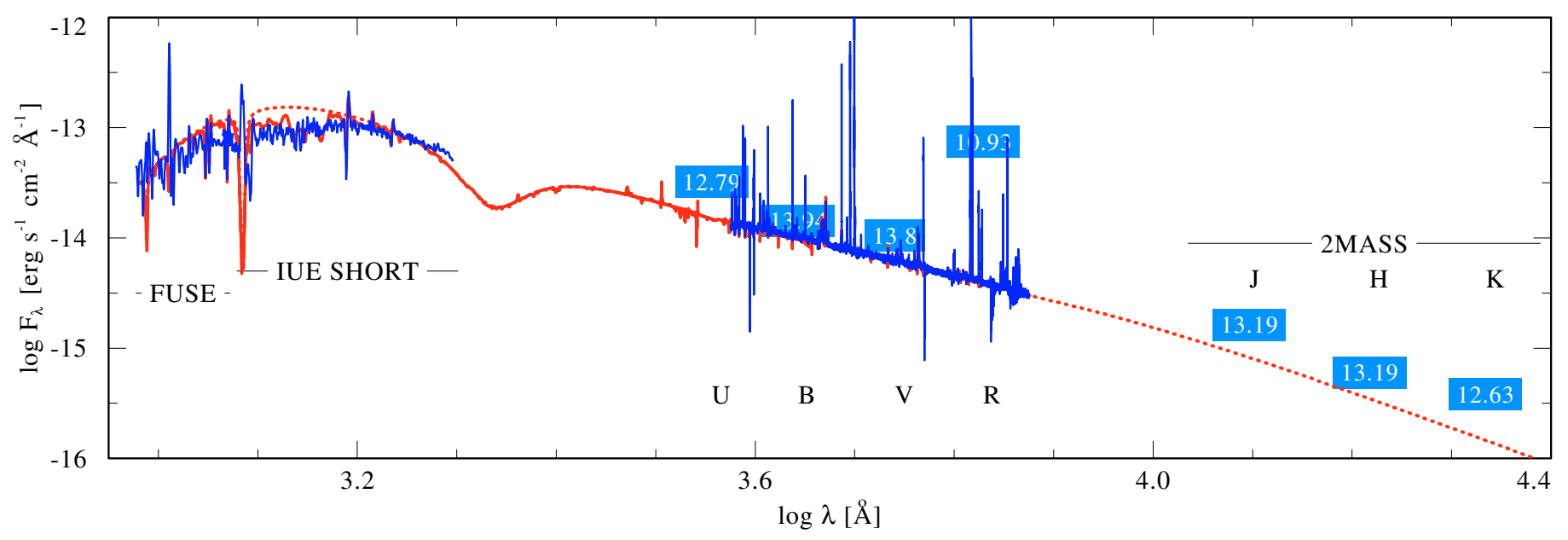

Fig. 7. Spectral energy distribution for CS PB 8, model vs. observation. Observed spectra (blue thin lines) are from FUSE, IUE, and MIKE (see Sect. 2). Photometric values (blue blocks) taken from Acker et al. (1992) for UBV, Space Telescope Science Institute \& Osservatorio Astronomico di Torino (2001) for $R$, and 2MASS (Skrutskie et al. 2006) for JHK are partly contaminated by nebular emission. The calculated spectrum (red line) is for the model parameters in Table 3. The model flux was reddened with $E_{B-V}=0.4$ and $R_{V}=4$ and corrected for interstellar Lyman line absorption. The model continuum without lines is also shown for comparison (red dotted). Note that in the IUE SHORT range the iron spectral lines form a pseudo-continuum.

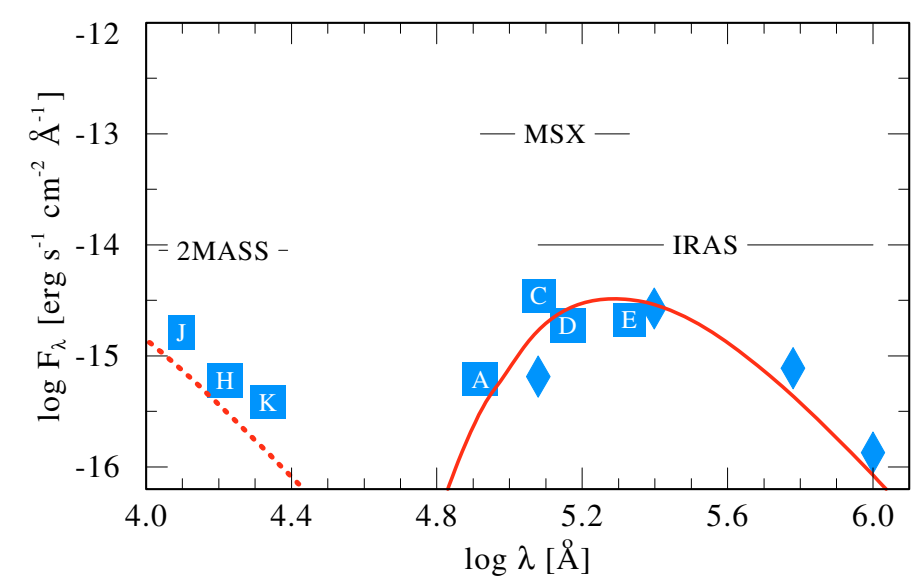

Fig. 8. SED for PB 8 in the infrared range. Photometric observations (blue blocks) for $J H K$ are from 2MASS, ACDE from MSX (MSXPSC V2.3), and 12, 25, 60, $100 \mu \mathrm{m}$ (blue diamonds) from IRAS (IPAC V2.0 1986). Also shown is the synthetic stellar continuum (red dotted) and a blackbody spectrum (red line) for $T=150 \mathrm{~K}$, indicating warm dust emission.

$R_{V}=3.1$. Following Cardelli et al. (1989), a higher value of $R_{V}$ can be interpreted as a larger dust grain size, meaning there may be different dust compositions along the line of sight towards the planetary nebula and the central star. A possible explanation could be dust within the nebula in the close vicinity of the central star, as there is a strong mid-IR emission visible in MSX and IRAS observations (Fig. 8). The comparison with a blackbody with $T=150 \mathrm{~K}$ indicates that this emission might be caused by "warm" dust. The 2MASS photometry values are higher than predicted by the stellar atmosphere model, presumably due to contamination by nebular emission.

With the adopted stellar luminosity of $6000 L_{\odot}$ we estimated a distance of $4.2 \mathrm{kpc}$ towards PB 8 . This value is of the same order of magnitude as the $5.15 \mathrm{kpc}$ which were derived from the nebular luminosity and its brightness temperature in the radio range $(5 \mathrm{GHz})$ and nebular distances, which lie between 2.2 and $5.8 \mathrm{kpc}$ (Phillips 2004, and references therein).
The observed FUSE flux cannot be reproduced consistently with the IUE and optical flux by any set of model parameters, distance or extinction. In particular, the flux between $1140 \AA$ and $1200 \AA$ seems to be too low compared with the model. This might be caused by problems with the channel alignment, as reported for other observations, e.g. Miksa et al. (2002). We checked the unbinned FUSE spectra from the program Z911, target 13 and found a discrepancy in the measured fluxes between the $2 \mathrm{ALiF}$ and $1 \mathrm{BLiF}$ channels of up to $50 \%$ in the range of $1140 \AA$ to $1200 \AA$. Therefore we applied a manual correction to the rebinned spectrum in this wavelength range (Fig. 9) to match the overlapping IUE flux.

FUSE spectra are usually affected by $\mathrm{H}_{2}$ absorption lines, nebular and airglow emission lines, which hamper the analysis of the underlying stellar spectrum. A simple fit of the $\mathrm{H}_{2}$ absorption lines by eye was performed with the help of adequate templates to tell interstellar from stellar absorption lines. For the fitting we used a column density of $n_{\mathrm{H}_{2}}(J=0)=4 \times 10^{19} \mathrm{~cm}^{-2}$ and excitation temperatures of $T_{J=0-J=1}=60 \mathrm{~K}$ and $T_{J=2-J=4}=270 \mathrm{~K}$. The synthetic stellar spectrum, which was corrected for this interstellar absorption from $\mathrm{H}_{2}$, is shown in Fig. 9. We found the same radial velocity of $v_{\text {rad }}=12 \mathrm{~km} \mathrm{~s}^{-1}$ for the central star, its planetary nebula, and the absorbing ISM.

To reproduce the observed $\mathrm{P}$ Cygni profiles of the O VI resonance doublet in the FUSE spectrum, super-ionization by X-ray emission was included in the model. For this purpose an optically thin hot gas component of $T=1.5 \mathrm{MK}$ was assumed to be distributed within the stellar wind. We only accounted for its free-free emission (thermal bremsstrahlung), because the filling factor was arbitrarily chosen (cf. Baum et al. 1992, for the formalism).

From our PoWR model (without additional X-ray emission) we predict an $\mathrm{HI}$ Zanstra temperature of $T(\mathrm{Hi})=53 \pm 3 \mathrm{kK}$. Shaw \& Kaler (1989) measured a lower value, $T(\mathrm{Hi})=32 \pm$ $3 \mathrm{kK}$. This discrepancy could mean that the nebula is not optically thick in all directions of the ionizing radiation. For He II the model predicts a Zanstra temperature of only $T(\mathrm{He}$ ii $)=$ $21 \pm 1 \mathrm{kK}$ due to the strong absorption in the helium-rich wind, which means that there should not be enough photons to create a noticeable zone of fully ionized helium in the nebula. This 


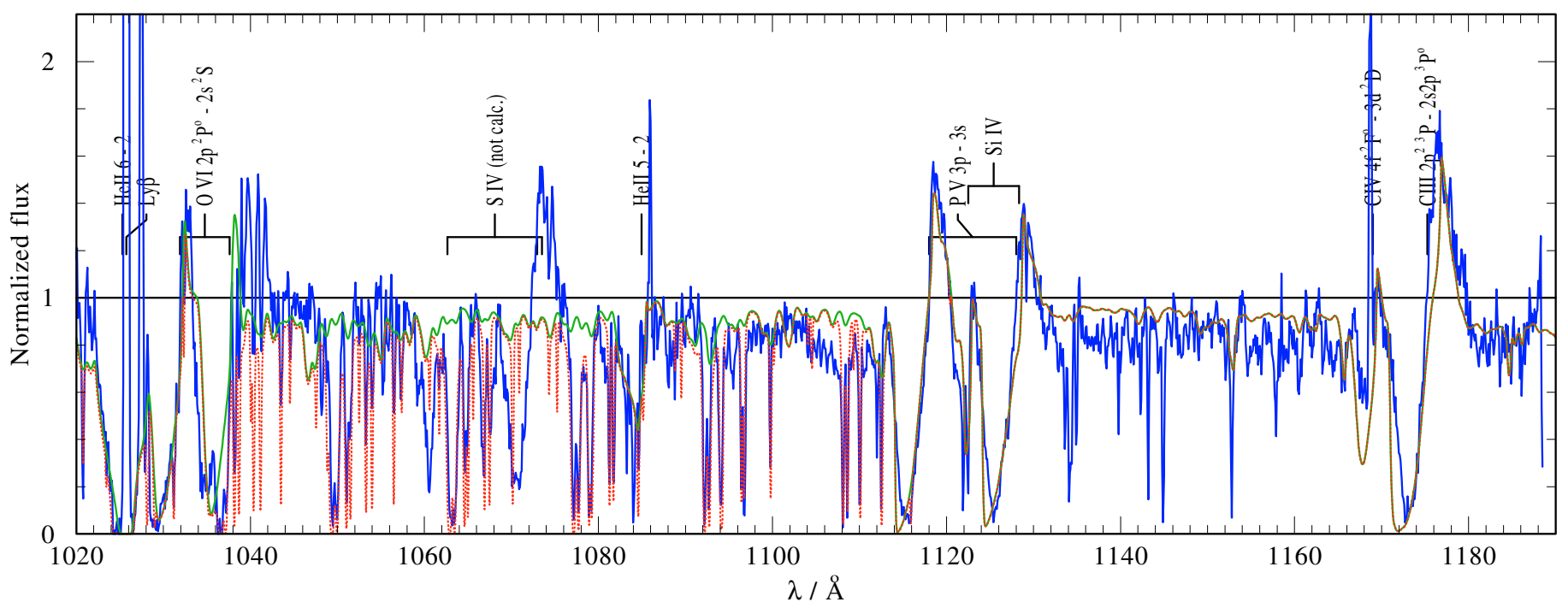

Fig. 9. Detail of the normalized FUSE spectrum (blue line) compared to the synthetic spectrum after accounting for interstellar $\mathrm{H}_{2}$ and Lyman absorption (red dotted). The stellar spectrum shows strong P Cygni profiles. Superimposed are narrow interstellar absorption lines of $\mathrm{H}_{2}$, emission lines from the planetary nebula, and telluric air glow features. The synthetic spectrum without interstellar $\mathrm{H}_{2}$ absorptions is shown for comparison (green).

agrees with García-Rojas et al. (2009), who could not detect any nebular He II lines in the spectrum of PB 8.

\subsection{Element abundances}

Hydrogen. He II lines from the Pickering series with even principle quantum numbers $n$ appear much stronger in the observation than the odd members of the same series. As the former are blended with the Balmer lines of hydrogen, this is clear evidence for a significant contribution from hydrogen. We obtained the best fit with a hydrogen mass fraction of $40 \%$.

Carbon. The carbon mass fraction derived from C III and C IV lines is only $X_{\mathrm{C}}=1.3 \%$. Spectral lines from other ionization stages, especially $\mathrm{C}$ II, are not detected.

Oxygen. The oxygen abundance is based on the emission lines from O III and O IV. The O VI resonance line at $1031 \AA$ in the FUSE spectrum depends on the super-ionization effect and is therefore not useful for the abundance determination.

Nitrogen. A nitrogen abundance of about $2 \%$ by mass is derived from the spectral lines of N III and N IV. The N V lines appear too weak in the model. Note that similar to O VI the excitation of $\mathrm{N} \mathrm{V}$ might be dominated by super-ionization.

Iron. Iron is included with a slightly super-solar abundance $\left(X_{\mathrm{Fe}}=1.6 \times 10^{-3}\right.$ by mass). For the iron-group elements $\mathrm{Sc}$, $\mathrm{Ti}, \mathrm{V}, \mathrm{Cr}, \mathrm{Mn}, \mathrm{Fe}, \mathrm{Co}$, and $\mathrm{Ni}$, a given relative abundance with respect to iron is adopted, as outlined in Gräfener et al. (2002). The iron forest, visible in the FUSE and IUE range below $1500 \AA$, is roughly reproduced with these abundances.

Phosphorus and silicon. With solar abundances, the $\mathrm{PV}$ and Si IV lines in the UV spectra are well fitted, see e.g. Fig. 2.

Stellar parameters and chemical abundances derived for the central star of PB 8 are compiled in Table 3.
Table 3. Parameters of PB 8.

\begin{tabular}{lrl}
\hline \hline$T_{*}$ & $52 \pm 2$ & $\mathrm{kK}$ \\
$v_{\infty}$ & $1000 \pm 100$ & $\mathrm{~km} \mathrm{~s}^{-1}$ \\
$\log \dot{M}$ & $-7.07_{-0.13}^{+0.17}$ & $M_{\odot} \mathrm{a}^{-1}$ \\
$\log R_{\mathrm{t}}$ & $1.43_{-0.08}^{+0.12}$ & $R_{\odot}$ \\
$E_{B-V}$ & $0.41 \pm 0.01$ & $\mathrm{mag}$ \\
$d\left(L_{*}=6000 L_{\odot}\right)$ & $4.2 \pm 0.2$ & $\mathrm{kpc}$ \\
$v_{\mathrm{rad}}$ & 12 & $\mathrm{~km} \mathrm{~s}^{-1}$ \\
$\mathrm{H}$ & $40_{-10}^{+20}$ & $\%$ mass fraction \\
$\mathrm{He}$ & $55_{-22}^{+11}$ & $\%$ mass fraction \\
$\mathrm{C}$ & $1.3_{-0.3}^{+0.7}$ & $\%$ mass fraction \\
$\mathrm{N}$ & $2.0_{-0.5}^{+1.0}$ & $\%$ mass fraction \\
$\mathrm{O}$ & $1.3_{-0.3}^{+0.7}$ & $\%$ mass fraction \\
$\mathrm{Fe}$ & $1.6 \times 10^{-3}$ & $\%$ mass fraction \\
$\mathrm{P}$ & $5.2 \times 10^{-6}$ & $\%$ mass fraction \\
$\mathrm{Si}$ & $3.2 \times 10^{-4}$ & $\%$ mass fraction \\
\hline
\end{tabular}

Note that the errors given there are inferred from the sequence of our PoWR models A, B, C.

\subsection{Nebula age}

For the nebula, García-Rojas et al. (2009) derived an expansion velocity of $14 \pm 2 \mathrm{~km} \mathrm{~s}^{-1}$ from the separation of the maxima of [O III] $\lambda 5007$. With the same method we found an expansion velocity of $19 \pm 6 \mathrm{~km} \mathrm{~s}^{-1}$ from [N II] $\lambda 6548$ and 6583 . Following Schönberner et al. (2005a), this kind of discrepancy is characteristic for young planetary nebulæ, as the individual emission lines form in different regions with different velocities in the beginning of the PN expansion. Especially for the determination of the kinematic age of the nebula, only the [N II] shell is a reliable indicator (Schönberner et al. 2005a). Therefore we estimated the shell radius from the [N II] spectrogram, obtaining a value of $2.5^{\prime \prime}$. No substructure of the [N II] doublet, which would indicate 

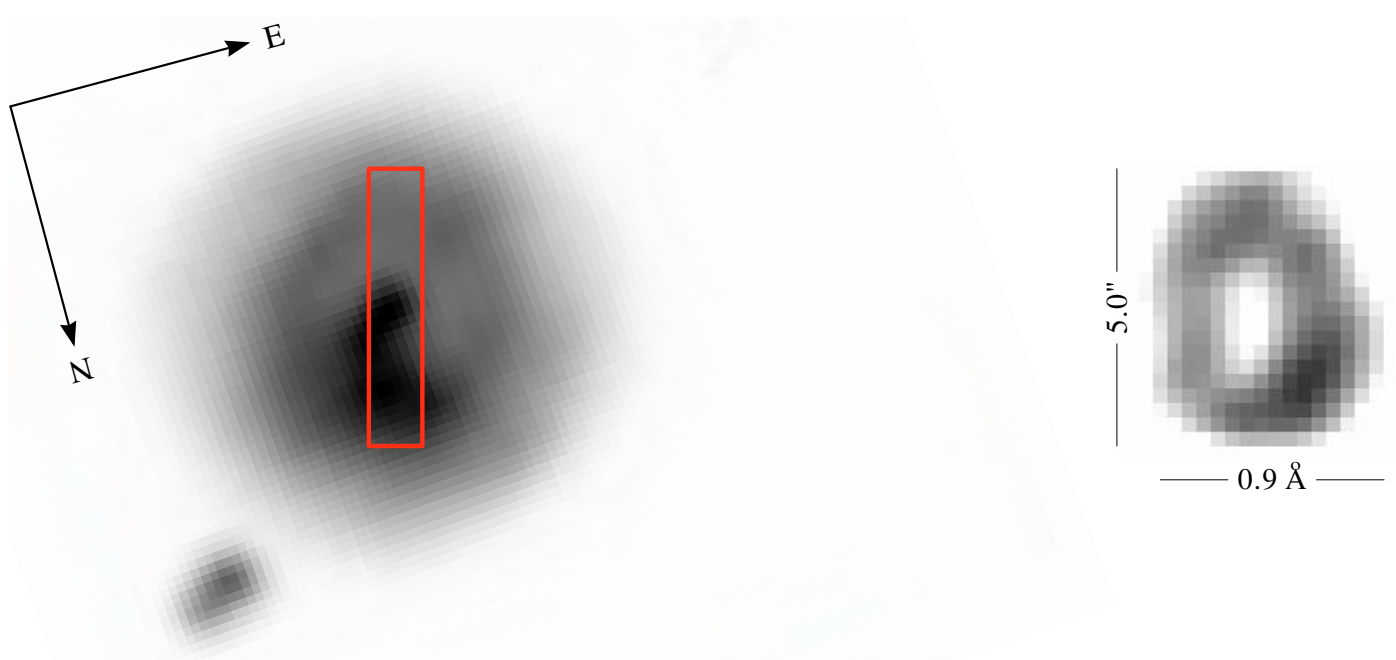

Fig. 10. Left: composite picture of PB 8 from $\mathrm{H} \alpha$, [N II], and [O III] emission lines with linear intensities (adapted from Schwarz et al. 1992). Also shown is the slit position (red rectangle) of our optical observation. The slit orientation was set to the parallactic angle, which actually varied between $-20^{\circ}$ and $-10^{\circ}$ during the exposure. The bright nebular knot in the northern part of the slit can also be recognized in the lower part of the spectrogram (cf. right panel). Right: spectrogram of the [O III] $5007 \AA$ line, indicating the roughly spherical symmetry of the nebula. This symmetry is seen along the slit as well as in the dispersion direction, which reflects the radial velocity along the line-of-sight. The southern end of the slit is to the top (cf. left panel).

different velocities for rim and shell, could be resolved. Thus we adopt the peak separation as the indicator for the shell expansion velocity and regard this as a lower limit. Together with the shell radius and the spectroscopic distance, this yields an upper limit for the dynamical age of the nebula of 2600 years, which agrees with Gesicki et al. (2006). Hence we conclude that PB 8 is a relatively young nebula.

\section{Discussion}

\subsection{PN and Central star status}

The nebula PB 8 appears as a roughly spherical nebula, nearly round in the composite image of $\mathrm{H} \alpha,[\mathrm{N} \mathrm{II}]$, and [O III] (Fig. 10, left panel $^{3}$ ), although the shell shows some knotty structure. In particular, there is a bright structure extending from the center to the northern side of the shell. The long-slit spectrogram also reveals a good symmetry in the radial velocities (Fig. 10, right panel). Given the unique chemical abundances of the central star PB 8, one must consider the possibility that this object is actually a massive star with a ring nebula. However, the low nebular expansion velocity discussed in Sect. 4.2 is rather characteristic for PNe. Medina et al. (2006) found expansion velocities for PNe with Wolf-Rayet nuclei in the range of $8-44 \mathrm{~km} \mathrm{~s}^{-1}$ from direct observations. Expansion velocities for ring nebulæ around massive stars are systematically higher, $16-110 \mathrm{~km} \mathrm{~s}^{-1}$ (Chu et al. 1999).

Moreover, the electron density in PB 8 measured by GarcíaRojas et al. (2009), $n_{\mathrm{e}}=2550 \pm 550 \mathrm{~cm}^{-3}$ is typical for young planetary nebulæ, but several times higher than found in ring nebulæ (Mathis et al. 1992).

Furthermore, if the central star of PB 8 were a massive star, this would imply a luminosity of at least $\log \left(L / L_{\odot}\right)=5.3$, which shifts the distance to $\approx 24.2 \mathrm{kpc}$. With a Galactic latitude of $4^{\circ}$ this corresponds to a height of $1.7 \mathrm{kpc}$ above the fundamental plane of the Galaxy. This is much more than the scale height of the thin disk and therefore an unlikely location for a massive star.

${ }^{3}$ From http://www.astro. washington.edu/balick/PNIC/

\subsection{Re-classification of the central star of $P B 8$}

The central star of PB 8 has been classified as spectral type [WC5-6] by Acker \& Neiner (2003). Yet we showed above that the central star of PB 8 is not a member of the [WC] sequence; its spectrum shows strong lines of nitrogen, reflecting that its chemical composition rather resembles that of a WN star. Nevertheless, carbon is slightly enhanced, in contrast to the typical $\mathrm{WN}$ composition where carbon is strongly depleted due to the CNO cycle equilibrium.

Among massive WR stars are a few objects with a similar composition as our program star, which are usually considered to be caught in the transition phase between the $\mathrm{WN}$ and $\mathrm{WC}$ stage. These are classified as spectral type WN/WC or WNC. Therefore in analogy to these massive stars we suggest to classify the central star of PB 8 as [WN/WC].

The detailed subtype of PB 8 is WN6 when applying the classification scheme established by Smith (1968) for massive WN stars. With the scheme in van der Hucht et al. (1981) for massive WC stars, the WC7 subtype seems to be appropriate. In combination with these two schemes, we determine the detailed subtype classification as [WN6/WC7].

The [WC5-6] classification of PB 8 by Acker \& Neiner (2003) was partly based on the identification of spectral features with stellar C II, but we cannot confirm any stellar C II line from our high-resolution data.

Tylenda et al. (1993) alternatively defined the class of "weak emission line stars" (WELS) for those spectra that show much fainter and narrower emission lines than massive WC stars. Gesicki et al. (2006) assign this WELS classification to PB 8. However, the nature and homogeneity of the WELS class seems to be still unclear.

There are two other known WR-type central stars with noncarbon-rich winds. One is LMC-N 66 in the LMC, which is only sometimes of the Wolf-Rayet type. It has an irregular nebula and seems to be a close binary (see discussion in Peña et al. 2004). The other example, the central star of PMR 5 discovered by Morgan et al. (2003), is probably a Galactic [WN] star. Its spectrum shows only helium and nitrogen lines, while any carbon lines are missing. In case of PB 8, carbon and oxygen lines 
are visible. Morgan et al. (2003) discuss the PN status of PMR 5 on the basis of the nebular expansion velocity and electron density. They conclude that PMR 5 is a normal PN.

\subsection{Evolutionary status}

The surface composition of PB 8 appears unique among all CSPNe that have been analyzed so far. Only two other CSPNe (PMR 5 and the enigmatic variable LMC-N 66) are known to show a WN-type composition, which is dominated by helium with a significant amount of nitrogen. Two more CSPNe are known to be helium-rich, but without strong winds (LoTr 4 and K 1-27, Rauch et al. 1998). Our program star PB 8 is unique in showing a significant amount of carbon, while carbon is usually depleted in WN-type compositions.

Note that there is a He-sdO star without PN, KS 292 (alias Hbg 292), that shows a similar composition as PB 8, including the enhanced carbon abundance (Rauch et al. 1991).

This poses the question of how to explain the evolutionary origin of PB 8 .

The formation of hydrogen-deficient post-AGB stars can be explained by a final thermal pulse which leads to the ingestion of the hydrogen envelope (Herwig et al. 1999; Herwig 2001; Werner \& Herwig 2006; Althaus et al. 2005). This final thermal pulse may occur either at the tip of the AGB (AGB final thermal pulse, AFTP) or later, when the AGB has been left (late thermal pulse, LTP, or very late thermal pulse, VLTP). These models lead to a carbon-rich surface composition (carbon abundance larger than $20 \%$ by mass), which is what is needed to explain the observed abundance patterns of [WC]-type central stars. However, the predicted nitrogen abundance is very small, except for the VLTP case where $X_{\mathrm{N}} \geq 1 \%$ has been predicted (Althaus et al. 2005; Werner \& Herwig 2006).

As a tentative explanation for PB 8, we propose that the final thermal pulse has only been "weak", so that only a small amount of carbon has been dredged up to the surface. The bulk of matter at the surface is then enriched by helium from the former intershell region. This material also contains nitrogen according to the equilibrium from the CNO cycle. In addition, a part of the hydrogen-rich envelope must have survived the last pulse and become mixed into the present outer layers. While it is not clear whether such a "weak" last thermal pulse can happen on the AGB, it might occur in an extremely late VLTP when the star is already too cool to undergo a full He-shell flash (F. Herwig, private communication).

Further constraints for the evolutionary origin of PB 8 may be derived from the planetary nebula. In Sect. 4.2 we showed that the present nebula is younger than 3000 years. There is no visible remnant of an older PN. Moreover, the nebula abundance ratios $\mathrm{He} / \mathrm{H}=0.123$ and $\mathrm{N} / \mathrm{O}=0.28$ by number (García-Rojas et al. 2009) show that PB 8 is not a helium-enriched Peimbert's Type I $\mathrm{PN}$. For the latter, $\mathrm{He} / \mathrm{H}>0.125$ or $\mathrm{N} / \mathrm{O}>0.5$ is expected (Peimbert \& Torres-Peimbert 1987). A VLTP origin of the nebula is therefore implausible. The low N/O ratio also indicates the absence of hot bottom burning (HBB), which is predicted for more massive AGB stars. From a comparison with stellar evolutionary tracks, Kaler \& Jacoby (1989) deduce N/O > 0.8 as a sharp limit for $\mathrm{N}$-enriched $\mathrm{PNe}$, which are supposed to indicate HBB in AGB-stars with $M_{\text {core }}>0.8 M_{\odot}$.

Thus the following alternative scenarios might explain our results:

1. The CSPN of PB 8 has a low mass and evolves slowly. For instance, a $0.6 M_{\odot}$ post-AGB star on the way to an LTP has a crossing time of 4000 years from $10^{4} \mathrm{~K}$ to its maximum effective temperature (Blöcker 2003). Then, either

(a) the present nebula was ejected by a born-again AGB-star after occurrence of a "weak" VLTP. A possible older PN from the first AGB phase has already dissolved. As mentioned above, this scenario does not fit well to PB 8, as the PN is not enriched in helium; or,

(b) the CSPN suffered an "anomalous AFTP", resulting in the observed surface abundances. The nebula was formed only during this AGB phase of the star. In this scenario it is difficult to explain the enhanced nitrogen abundance of the star, as nitrogen enrichment is neither predicted for the AFTP nor can it originate from HBB.

2. Alternatively, the CS of PB 8 may have a relatively high mass and therefore may have evolved very fast. The crossing time for e.g. a $0.94 M_{\odot}$ post-AGB star is only 50 years (Blöcker 2003). A VLTP has already occurred, but most of the nebula observed now still originates from the first AGB period, not from the born-again AGB star after the VLTP. Albeit possible, this scenario has a low probability because the empirical mass distribution of central stars has a sharp maximum at $0.6 M_{\odot}$ and declines substantially towards higher values (Tylenda 2003); furthermore there are no hints of HBB, which would be indicative for a more massive CSPN.

However, one has to keep in mind that the appropriate stellar evolution models are still calculated only in 1D. Especially the convective mixing during $\mathrm{H}$-ingestion flashes of the TPs were treated in diffusion approximation, whereas recent hydrodynamical studies, e.g. by Woodward et al. (2008), emphasize that convective mixing is rather an advection process, making $2 \mathrm{D}$ or $3 \mathrm{D}$ calculations necessary.

Alternatively to single star evolution, one may consider binarity with a common envelope phase as the origin of the hydrogen deficiency. However, PB 8 shows no evidence of binarity. The nebula does not look bipolar. Also, Méndez (1989) found no indication of radial velocity variations between three spectra taken within one year, but only changes of the P-Cygni line profiles, which must be attributed to variability of the stellar wind.

Summarizing, the evolutionary origin of PB 8 cannot be explained by any existing model for a post-AGB star which lost its hydrogen envelope in a final thermal pulse. However, one can imagine scenarios of a weak or anomalous thermal pulse, occurring on the AGB or later, which may explain the unique chemical composition of this star and its young nebula.

The chemical composition found in the expanding atmosphere of the central star of PB 8 differs from any known central star abundance. However, it resembles the rare transition class of WN/WC subtypes of massive Wolf-Rayet stars. Therefore we suggest to open a new class of [WN/WC]-type central stars with PB 8 as its first member.

Acknowledgements. M. Peña acknowledges financial support from FONDAPChile and DGAPA-UNAM (grants IN118405 and IN112708). This work was supported by the Bundesministerium für Bildung und Forschung (BMBF) under grant 05AVIPB/1. M. Peña is grateful to the Institute for Physics and Astronomy, Potsdam University, for hospitality and financial support when part of this work was done.

\section{References}

Acker, A., \& Neiner, C. 2003, A\&A, 403, 659

Acker, A., Marcout, J., Ochsenbein, F., Stenholm, B., \& Tylenda, R. 1992, Strasbourg - ESO catalogue of galactic planetary nebulae, Part 1, Part 2 (Garching: European Southern Observatory) 
Althaus, L. G., Serenelli, A. M., Panei, J. A, et al. 2005, A\&A, 435, 631

Baum, E., Hamann, W.-R., Koesterke, L., \& Wessolowski, U. 1992, A\&A, 266, 402

Blöcker, T. 2003, in Planetary Nebulae: Their Evolution and Role in the Universe, ed. S. Kwok, M. Dopita, \& R. Sutherland, IAU Symp., 209, 101

Cardelli, J. A., Clayton, G. C., \& Mathis, J. S. 1989, ApJ, 345, 245

Chu, Y.-H., Weis, K., \& Garnett, D. R. 1999, AJ, 117, 1433

Crowther, P. A. 2008, in Hydrogen-Deficient Stars, ed. A. Werner, \& T. Rauch, ASP Conf. Ser., 391, 83

García-Rojas, J., Peña, M., \& Peimbert, A. 2009, A\&A, 496, 139

Gesicki, K., Zijlstra, A. A., Acker, A., et al. 2006, A\&A, 451, 925

Gräfener, G., Koesterke, L., \& Hamann, W.-R. 2002, A\&A, 387, 244

Groenewegen, M. A. T., \& Lamers, H. J. G. L. M. 1989, A\&AS, 79, 359

Hamann, W.-R., \& Gräfener, G. 2004, A\&A, 427, 697

Hamann, W.-R., \& Koesterke, L. 1998, A\&A, 335, 1003

Herwig, F. 2001, Ap\&SS, 275, 15

Herwig, F., Blöcker, T., Langer, N., \& Driebe, T. 1999, A\&A, 349, L5

Hillier, D. J., \& Miller, D. L. 1999, ApJ, 519, 354

Kaler, J. B., \& Jacoby, G. H. 1989, ApJ, 345, 871

Koesterke, L. 2001, Ap\&SS, 275, 41

Mathis, J. S., Cassinelli, J. P., van der Hucht, K. A., et al. 1992, ApJ, 384, 197

McCandliss, S. R. 2003, PASP, 115, 651

Medina, S., Peña, M., Morisset, C., \& Stasińska, G. 2006, Rev. Mex. Astron. Astrofis., 42, 53

Méndez, R. H. 1989, in Planetary Nebulae, ed. S. Torres-Peimbert, IAU Symp., 131,261

Méndez, R. H. 1991, in Evolution of Stars: the Photospheric Abundance Connection, ed. G. Michaud, \& A. V. Tutukov, IAU Symp., 145, 375

Miksa, S., Deetjen, J. L., Dreizler, S., et al. 2002, A\&A, 389, 953
Miller Bertolami, M. M., \& Althaus, L. G. 2007, MNRAS, 380, 763 Morgan, D. H., Parker, Q. A., \& Cohen, M. 2003, MNRAS, 346, 719 Paczyński, B. 1970, Acta Astronomica, 20, 47

Peña, M., Hamann, W.-R., Ruiz, M. T., Peimbert, A., \& Peimbert, M. 2004, A\&A, 419, 583

Peimbert, M., \& Torres-Peimbert, S. 1987, Rev. Mex. Astron. Astrofis., 14, 540 Phillips, J. P. 2004, MNRAS, 353, 589

Rauch, T., Dreizler, S., \& Wolff, B. 1998, A\&A, 338, 651

Rauch, T., Heber, U., Hunger, K., Werner, K., \& Neckel, T. 1991, A\&A, 241, 457

Schmutz, W., Hamann, W.-R., \& Wessolowski, U. 1989, A\&A, 210, 236

Schönberner, D. 1989, in Planetary Nebulae, ed. S. Torres-Peimbert, IAU Symp., 131,463

Schönberner, D., Jacob, R., \& Steffen, M. 2005a, A\&A, 441, 573

Schönberner, D., Jacob, R., Steffen, M., et al. 2005b, A\&A, 431, 963

Schwarz, H. E., Corradi, R. L. M., \& Melnick, J. 1992, A\&AS, 96, 23

Shaw, R. A., \& Kaler, J. B. 1989, ApJS, 69, 495

Skrutskie, M. F., Cutri, R. M., Stiening, R., et al. 2006, AJ, 131, 1163

Smith, L. F. 1968, MNRAS, 138, 109

Space Telescope Science Institute \& Osservatorio Astronomico di Torino 2001, VizieR Online Data Catalog, 1271

Tylenda, R. 2003, in Planetary Nebulae: Their Evolution and Role in the Universe, ed. S. Kwok, M. Dopita, \& R. Sutherland, IAU Symp., 209, 159

Tylenda, R., Acker, A., \& Stenholm, B. 1993, A\&AS, 102, 595

van der Hucht, K. A., Conti, P. S., Lundstrom, I., \& Stenholm, B. 1981, Space Sci. Rev., 28, 227

Werner, K., \& Herwig, F. 2006, PASP, 118, 183

Woodward, P., Herwig, F., Porter, D., et al. 2008, in First Stars III, ed. B. W. O’Shea, \& A. Heger, American Institute of Physics Conf. Ser., 990, 300 